\title{
Distribución geográfica y estado de conocimiento de las especies del género Tamandua (Xenarthra: Myrmecophagidae) en Colombia
}

\author{
Melissa Alzate-Gaviria ${ }^{\mathrm{A}, \mathrm{B}, 1}$, José Fernando GonzÁlez-Maya ${ }^{\mathrm{C}}$ y Álvaro Botero-Botero ${ }^{\mathrm{D}}$
}

\author{
AFundación AIUNAU, Calima Mza 11 \#4, Armenia, Quindío, Colombia. E-mail: dayanaal_1108@hotmail.com \\ ${ }^{B}$ Programa de Biología, Facultad de Ciencias Básicas, Universidad del Quindío, Carrera 15 \#12 Norte, Armenia, Quindío, Colombia. \\ 'Proyecto de Conservación de Aguas y Tierras (ProCat), Carrera 13 No. 96-82 Oficina 205, Bogotá, Cundinamarca, Colombia. \\ E-mail: jfgonzalezmaya@gmail.com \\ D Programa de Licenciatura en Biología, Universidad del Quindío, Carrera 15 \#12 Norte, Armenia, Quindío, Colombia. \\ E-mail: albotero33@yahoo.com
}

${ }^{1}$ Autor para correspondencia

\begin{abstract}
Resumen La escasa información científica que existe del género Tamandua en Colombia resulta en poco interés hacia estas especies. Por esta razón, este artículo presenta una compilación y análisis de información para establecer la distribución geográfica, estado de conocimiento y prioridades de investigación de las especies de este género en Colombia. Los datos recabados indican que su distribución abarca casi la totalidad del territorio colombiano, encontrándose en todas las regiones biogeográficas del país. Tamandua mexicana se distribuye en las regiones Caribe, el Pacífico y los Andes, y T. tetradactyla en la Orinoquia, la Amazonia y en la Cordillera Central. Se registra una posible zona de simpatría entre las dos especies en los departamentos de Cundinamarca, Huila, Valle del Cauca, Caldas y Quindío, por lo que es necesario realizar estudios que corroboren dicho solapamiento. En total se encontraron siete estudios puntuales sobre el género, lo que evidencia un estado del conocimiento pobre. Estos se basaron principalmente en la dieta y presencia de ectoparásitos. Se espera que con estos resultados se lograre instaurar prioridades de investigación que abarquen aspectos biológicos y ecológicos de estos hormigueros en Colombia.
\end{abstract}

Palabras clave: ecología, hormigueros, región biogeográfica, simpatría

Geographic distribution and state of knowledge of species of the genus Tamandua (Xenarthra: Myrmecophagidae) in Colombia

Abstract The limited scientific information available for the genus Tamandua in Colombia results in little interest towards these species. For this reason, this article presents a compilation and analysis of information to establish the geographic distribution, state of knowledge, and research priorities of the species of this genus in Colombia. The collected data indicate that their distribution includes almost the entire Colombian territory and all biogeographic regions of the country. Tamandua mexicana is distributed in Caribe, Pacífico, and Andes regions, and T. tetradactyla in Orinoquia, Amazonia, and the Central Cordillera. We registered an area of possible sympatry between the two species in the departments of Cundinamarca, Huila, Valle del Cauca, Caldas, and Quindío. We found seven specific studies, which indicates a state of poor knowledge. These studies were mainly based on their diet and the presence of ectoparasites. We hope these results will promote interest in establishing research priorities that cover biological and ecological aspects of these anteaters in Colombia.

Keywords: anteaters, biogeographic region, ecology, sympatry 


\section{INTRODUCCIÓN}

El orden Pilosa incluye al género Tamandua que se encuentra conformado por las especies T. mexicana y T. tetradactyla (Wetzel, 1985). Son especies de hábitos diurnos o crepusculares y solitarias (Wetzel, 1985; Miranda et al., 2014; Ortega Reyes et al., 2014). Estas especies poseen un amplio rango de distribución. Tamandua mexicana se puede encontrar en México, Guatemala, El Salvador, Honduras, Costa Rica, Panamá, Colombia, noroccidente de Venezuela, Ecuador y noroccidente de Perú y T. tetradactyla desde Colombia, Venezuela, Brasil, Bolivia, oriente de Ecuador y Perú hasta el norte de Argentina (Gardner, 2008; Miranda et al., 2014). En el territorio colombiano, T. mexicana ocurre en el norte, occidente y centro del país y T. tetradactyla en el suroriente. Ambas especies se pueden encontrar en bosques secundarios, bosques húmedos tropicales, sabanas y manglares, en alturas que van desde 0 hasta los $2.000 \mathrm{msnm}$ (Alberico et al., 2000).

Las tamandúas están clasificadas como "Preocupación Menor" (LC) a nivel global por su amplio rango de distribución (UICN, 2016). En Colombia, se les incluye en esta misma categoría. Sin embargo, debido a que no se cuenta con el conocimiento necesario, se desconoce el alcance que puedan tener las diferentes perturbaciones antrópicas sobre sus poblaciones (Meisel, 2003; Alves da Rosa, 2010; Superina et al., 2010).

Por lo anterior, el objetivo de este estudio fue determinar el estado actual del conocimiento del género Tamandua en cuanto a los principales aspectos biológicos, ecológicos, de conservación y su distribución en Colombia. Se incluyó la presencia del género en áreas protegidas, con el fin de identificar las prioridades de investigación a nivel temático y espacial para las dos especies presentes en el país.

\section{MATERIALES Y MÉTODOS}

Inicialmente se realizó una revisión bibliográfica consultando bases de datos y revistas científicas nacionales e internacionales para establecer el estado del conocimiento y distribución del género en Colombia. Para complementar la información sobre la distribución, fueron revisadas las bases de datos de diferentes museos de ciencias naturales e institutos nacionales e internacionales como The Field Museum of Chicago, Museum of Vertebrate Zoology, Smithsonian Institution National Museum of Natural History, Instituto Alexander Von Humboldt, Museo de Ciencias Naturales La Salle, Instituto de Ciencias Naturales Universidad Nacional, Museo Departamental de Ciencias Naturales Federico Carlos Lehmann y Museo de Historia Natural Universidad del Cauca. Durante esta revisión se documentó para cada registro el departamento de origen, fecha de ingreso del espécimen y colector.
Adicionalmente, se realizaron entrevistas a expertos nacionales y extranjeros incluyendo miembros del Grupo de Especialistas en Osos Hormigueros, Perezosos y Armadillos de la IUCN/SSC, la Sociedad de Mastozoología de Colombia, la Asociación Colombiana de Zoología y personas vinculadas a universidades y centros de investigación relacionadas con la temática (APÉndice 1). Por último, se contactaron 31 Corporaciones Autónomas Regionales (CAR) del país, buscando registros del género en sus jurisdicciones con el fin de obtener una distribución más completa sobre las tamandúas en Colombia. De seis CAR no se pudo obtener ningún tipo de información ya que no respondieron a la solicitud.

La bibliografía encontrada se analizó teniendo en cuenta la fecha de publicación, área de estudio (especificando los departamentos y municipios) y aspectos biológicos puntuales evaluados, con el fin de establecer prioridades de investigación en el país. Finalmente, por medio del programa ArcGis 10.3 (Environmental Systems Research Institute, Redlands, CA, Estados Unidos) fue generado un mapa de distribución de las especies utilizando todos los registros obtenidos durante la recopilación de información. Se ubicaron los puntos de presencia en el Sistema Nacional de Áreas Protegidas (SINAP) del país y se especificaron los sitios con vacíos de información y las zonas de posible simpatría entre las especies.

\section{RESULTADOS}

\section{Estado del conocimiento}

En total se obtuvieron 35 documentos, de los cuales 17 hacían referencia a T. mexicana y 12 a T. tetradactyla, cinco incluían ambas especies y solo en uno la especie no fue identificada. Los documentos se clasificaron en tres tesis de pregrado, 10 listados e informes presentados a entidades gubernamentales y privadas, un manual de rehabilitación, un programa para la conservación y 20 artículos científicos (TABLA 1). Los temas principales de los estudios fueron: listados de mamíferos, aprovechamiento de fauna, microbiología, estudios hematológicos, dieta y ectoparásitos, y estaban enfocados a departamentos o sitios específicos del país. Los listados de mamíferos y los documentos donde se utilizó material biológico de los individuos (heces, contenido estomacal, muestras de sangre) por medio de capturas y avistamientos, fueron los que aportaron la mayor información sobre la distribución de las especies en Colombia. Siete documentos son producciones bibliográficas exclusivas para el género; estos estudios estuvieron enfocados en análisis de ectoparásitos ( $T$. tetradactyla: Torres-Mejía et al., 2006; Gómez et al., 2010; RojanoBolaño et al., 2015), dieta y microbiología (T. mexicana: Vásquez et al., 2013; Triana-Ardila et al., 2011; T. tetradactyla: Sandoval-Gómez et al., 2012) y hematología (T. mexicana: Padilla-Cordero et al., 2014), realizados entre el 2006 y el 2015 en los departamentos del Valle del 
TABLA 1. Producción bibliográfica publicada sobre el género Tamandua en Colombia. En negrita figuran los estudios exclusivos para la especie.

\begin{tabular}{|c|c|c|c|c|c|}
\hline Año & Especie & $\begin{array}{c}\text { Localidad } \\
\text { (Departamento) }\end{array}$ & Tipo de registro & Tipo de documento & Fuente \\
\hline 2004 & Tamandua sp. & $\begin{array}{l}\text { Reserva Río Blanco } \\
\text { (Caldas) }\end{array}$ & Entrevista a comunidad & Listado de mamíferos & Sánchez et al., 2004 \\
\hline 2003 & T. mexicana & (Antioquia) & Espécimen en museo & Listado de mamíferos & $\begin{array}{c}\text { Cuartas-Calle \& } \\
\text { Muñoz-Arango, } 2003\end{array}$ \\
\hline 2003 & T. mexicana & $\begin{array}{c}\text { Manizales, Samaná } \\
\text { (Caldas) }\end{array}$ & Espécimen en museo & Listado de mamíferos & Castaño et al., 2003 \\
\hline 2006 & T. mexicana & $\begin{array}{c}\text { Vereda Aguas Regadas } \\
\text { (Bolívar) }\end{array}$ & Heces & $\begin{array}{l}\text { Informe para entidad } \\
\text { gubernamental }\end{array}$ & $\begin{array}{l}\text { Ayerbe-Quiñones et al., } \\
2006\end{array}$ \\
\hline 2007 & T. mexicana & Tutunendo (Chocó) & $\begin{array}{l}\text { Avistamiento/ } \\
\text { Entrevista a comunidad }\end{array}$ & Artículo científico & Cuesta-Ríos et al., 2007 \\
\hline 2010 & T. mexicana & Nariño & Espécimen en museo & Listado de mamíferos & $\begin{array}{c}\text { Ramírez-Chaves \& } \\
\text { Noguera-Urbano, } 2010\end{array}$ \\
\hline 2010 & T. mexicana & $\begin{array}{l}\text { PNN Isla Salamanca } \\
\text { (Magdalena) }\end{array}$ & Avistamiento & Artículo científico & $\begin{array}{l}\text { Balaguera-Reina et al., } \\
\qquad 2010\end{array}$ \\
\hline 2010 & T. mexicana & Río Patía (Cauca) & Avistamiento & Artículo científico & $\begin{array}{l}\text { Ramírez-Chaves et al., } \\
2010\end{array}$ \\
\hline 2010 & T. mexicana & Sucre & Avistamiento & $\begin{array}{l}\text { Informe para entidad } \\
\text { gubernamental }\end{array}$ & Galván-Guevara, 2010 \\
\hline 2010 & T. mexicana & Río La Miel (Caldas) & Ornamento & Articulo científico & $\begin{array}{c}\text { Castaño \& Corrales, } \\
2010\end{array}$ \\
\hline 2010 & T. mexicana & $\begin{array}{l}\text { Corredor Biológico San- } \\
\text { tuario de Fauna y Flora } \\
\text { Los Colorados - San- } \\
\text { tuario de Fauna y Flora } \\
\text { el Corchal "El Mono } \\
\text { Hernández" (Sucre) }\end{array}$ & Avistamientos & Artículo científico & $\begin{array}{c}\text { Castaño-Uribe et al., } \\
2010\end{array}$ \\
\hline 2011 & T. mexicana & $\begin{array}{l}\text { Área Natural Única } \\
\text { Los Estoraques- Ocaña } \\
\text { (Norte de Santander) }\end{array}$ & Captura (Dieta) & Tesis de pregrado & Triana-Ardila et al., 2011 \\
\hline 2013 & T. mexicana & Pedraza (Magdalena) & Entrevista & Artículo científico & Rojano et al., 2013 \\
\hline 2013 & T. mexicana & Magdalena y Bolívar & Captura (Microbiología) & Artículo científico & Vásquez et al., 2013 \\
\hline 2014 & T. mexicana & Córdoba y Magdalena & $\begin{array}{l}\text { Captura (Estudios } \\
\text { hematológicos) }\end{array}$ & Artículo científico & $\begin{array}{l}\text { Padilla-Cordero et al., } \\
2014\end{array}$ \\
\hline 2015 & T. mexicana & $\begin{array}{l}\text { Toluviejo - La Caimane- } \\
\text { ra (Sucre) }\end{array}$ & $\begin{array}{c}\text { Avistamiento } \\
\text { (Atropellamiento) }\end{array}$ & Artículo científico & $\begin{array}{c}\text { De La Ossa-V. \& Galván } \\
\text { Guevara, } 2015\end{array}$ \\
\hline 2015 & T. mexicana & Macanal (Boyacá) & $\begin{array}{c}\text { Avistamiento } \\
\text { (Cámara trampa) }\end{array}$ & $\begin{array}{l}\text { Informe para entidad } \\
\text { gubernamental }\end{array}$ & CORPOCHIVOR, 2015 \\
\hline 2015 & T. mexicana & Córdoba y Sucre & Decomisos CAR & Artículo científico & $\begin{array}{l}\text { Humanez-López } \\
\text { et al., } 2015\end{array}$ \\
\hline 1998 & T. tetradactyla & Orinoco & Revisión & Artículo científico & $\begin{array}{c}\text { Defler \& Rodríguez-Ma- } \\
\text { hecha, } 1998\end{array}$ \\
\hline 1999 & T. tetradactyla & $\begin{array}{l}\text { PNN La Paya } \\
\text { (Putumayo) }\end{array}$ & Entrevista a comunidad & Artículo científico & $\begin{array}{c}\text { Polanco-Ochoa et al., } \\
1999\end{array}$ \\
\hline 2001 & T. tetradactyla & $\begin{array}{l}\text { RNN Nukak } \\
\text { (Guaviare) }\end{array}$ & $\begin{array}{l}\text { Entrevista a comunidad } \\
\quad \text { - Avistamiento }\end{array}$ & $\begin{array}{c}\text { Informe para entidad } \\
\text { privada }\end{array}$ & Etter, 2001 \\
\hline
\end{tabular}




\begin{tabular}{|c|c|c|c|c|c|}
\hline Año & Especie & $\begin{array}{c}\text { Localidad } \\
\text { (Departamento) }\end{array}$ & Tipo de registro & Tipo de documento & Fuente \\
\hline 2004 & T. tetradactyla & $\begin{array}{l}\text { Río Guaviare } \\
\text { (Guaviare) }\end{array}$ & Avistamiento & $\begin{array}{l}\text { Informe para entidad } \\
\text { gubernamental }\end{array}$ & Romero, 2004 \\
\hline 2006 & T. tetradactyla & Quindío & Captura (Ectoparásitos) & Artículo científico & Torres-Mejía et al., 2006 \\
\hline 2007 & T. tetradactyla & $\begin{array}{l}\text { Vereda Barracones } \\
\text { (Arauca) }\end{array}$ & Avistamiento & Tesis de pregrado & $\begin{array}{l}\text { De Armas-Mendoza \& } \\
\text { Forero-Medina, } 2007\end{array}$ \\
\hline 2007 & T. tetradactyla & Casanare & $\begin{array}{l}\text { Revisión y recopilación } \\
\text { de información }\end{array}$ & Artículo científico & Vega-Orduz, 2007 \\
\hline 2009 & T. tetradactyla & Río Inírida (Vichada) & Avistamiento & Artículo científico & Ferrer-Pérez et al., 2009 \\
\hline 2010 & T. tetradactyla & $\begin{array}{l}\text { RNN Yotoco (Valle del } \\
\text { Cauca) }\end{array}$ & Captura (Ectoparásitos) & Artículo científico & Gomez et al., 2010 \\
\hline 2011 & T. tetradactyla & La Ceiba (Guainía) & $\begin{array}{l}\text { Encuestas y recopilación } \\
\text { de información }\end{array}$ & Tesis de pregrado & Cruz \& Ramos, 2011 \\
\hline 2015 & T. tetradactyla & El Amaparo (Casanare) & $\begin{array}{c}\text { Captura } \\
\text { (Microbiología) }\end{array}$ & Artículo científico & Rendón et al., 2015 \\
\hline 2015 & T. tetradactyla & Pore (Casanare) & $\begin{array}{c}\text { Captura } \\
\text { (Ectoparásitos) }\end{array}$ & Artículo científico & $\begin{array}{l}\text { Rojano-Bolaño et al., } \\
2015\end{array}$ \\
\hline 2000 & $\begin{array}{l}\text { T. mexicana } \\
\text { T. tetradactyla }\end{array}$ & $\begin{array}{c}\text { Regiones Andes, Caribe, } \\
\text { Pacífico } \\
\text { Regiones Amazonas, } \\
\text { Orinoco, Andes }\end{array}$ & Espécimen en museo & Listado de mamíferos & Alberico et al., 2000 \\
\hline 2012 & $\begin{array}{l}\text { T. mexicana } \\
\text { T. tetradactyla }\end{array}$ & $\begin{array}{l}\text { Maceo (Antioquia) } \\
\text { (Meta) }\end{array}$ & Avistamientos (Dieta) & Artículo científico & $\begin{array}{l}\text { Sandoval-Gómez } \\
\text { et al., } 2012\end{array}$ \\
\hline 2013 & $\begin{array}{l}\text { T. mexicana } \\
\text { T. tetradactyla }\end{array}$ & Colombia & Espécimen museo & Listado de mamíferos & Solari et al., 2013 \\
\hline 2014 & $\begin{array}{l}\text { T. mexicana } \\
\text { T. tetradactyla }\end{array}$ & Colombia & $\begin{array}{l}\text { Recopilación de } \\
\text { información }\end{array}$ & $\begin{array}{l}\text { Manual de } \\
\text { Rehabilitación }\end{array}$ & Rojano et al., 2014 \\
\hline 2016 & $\begin{array}{l}\text { T. mexicana } \\
\text { T. tetradactyla }\end{array}$ & Colombia & $\begin{array}{l}\text { Recopilación de } \\
\text { información }\end{array}$ & $\begin{array}{c}\text { Programa Nacional } \\
\text { para la conservación de } \\
\text { Xenarthras }\end{array}$ & $\begin{array}{l}\text { AIUNAU \& Minam- } \\
\text { biente, } 2016\end{array}$ \\
\hline
\end{tabular}

Cauca, Quindío, Norte de Santander, Meta, Antioquia, Córdoba, Magdalena y Casanare (TABLA 1). La recolección de material biológico para el desarrollo de estas investigaciones se obtuvo de individuos capturados en áreas protegidas, decomisados por CAR's o encontrados por atropellamientos en carreteras del país.

\section{Distribución geográfica}

En total se obtuvieron 344 registros del género en Colombia. De estos, 183 corresponden a registros en colecciones biológicas, tanto nacionales como internacionales, de especímenes colectados desde el año 1898 hasta 1997. En estos registros se encontraron 136 reportes para T. mexicana, 40 para T. tetradactyla y siete se encontraron sin identificar (Tamandua sp.). Los 161 registros restantes provienen de datos obtenidos a partir de entrevistas a expertos, consultas a las CAR's, búsquedas en bases de datos y revistas nacionales e internacionales. De estos, 135 son de T. mexicana, 25 de T. tetradactyla y un registro donde la especie se encontraba sin identificar (Tamandua sp.).
De acuerdo a estos registros T. mexicana se distribuye por el occidente y centro del país, encontrándose en las zonas biogeográficas del Caribe, el Pacífico y los Andes. La especie está presente en los departamentos de La Guajira, Santander, Sucre, Bolívar, Córdoba, Cesar, Atlántico, Norte de Santander, Magdalena, Chocó, Antioquia, Cauca, Valle del Cauca, Risaralda, Caldas, Quindío, Cundinamarca, Boyacá, Tolima, Huila y Nariño. Por su parte, T. tetradactyla se distribuye en el suroriente del país. Esta especie se pudo encontrar en las zonas biogeográficas de la Orinoquia y la Amazonia, incluyendo los departamentos de Vichada, Caquetá, Meta, Arauca, Guainía, Casanare, Guaviare, Vaupés, Putumayo y Amazonas. También se encontraron registros de T. tetradactyla en la región biogeográfica de los Andes, el Pacífico y un registro en el Caribe colombiano, pero no es claro si estos individuos fueron identificados correctamente o fueron traficados a estas regiones. Los registros de Tamandua sp. se ubicaron en la zona central de país, en los departamentos de Antioquia, Cundinamarca y Meta (FIG. 1). $\mathrm{Al}$ sobreponer los registros de distribución sobre el 


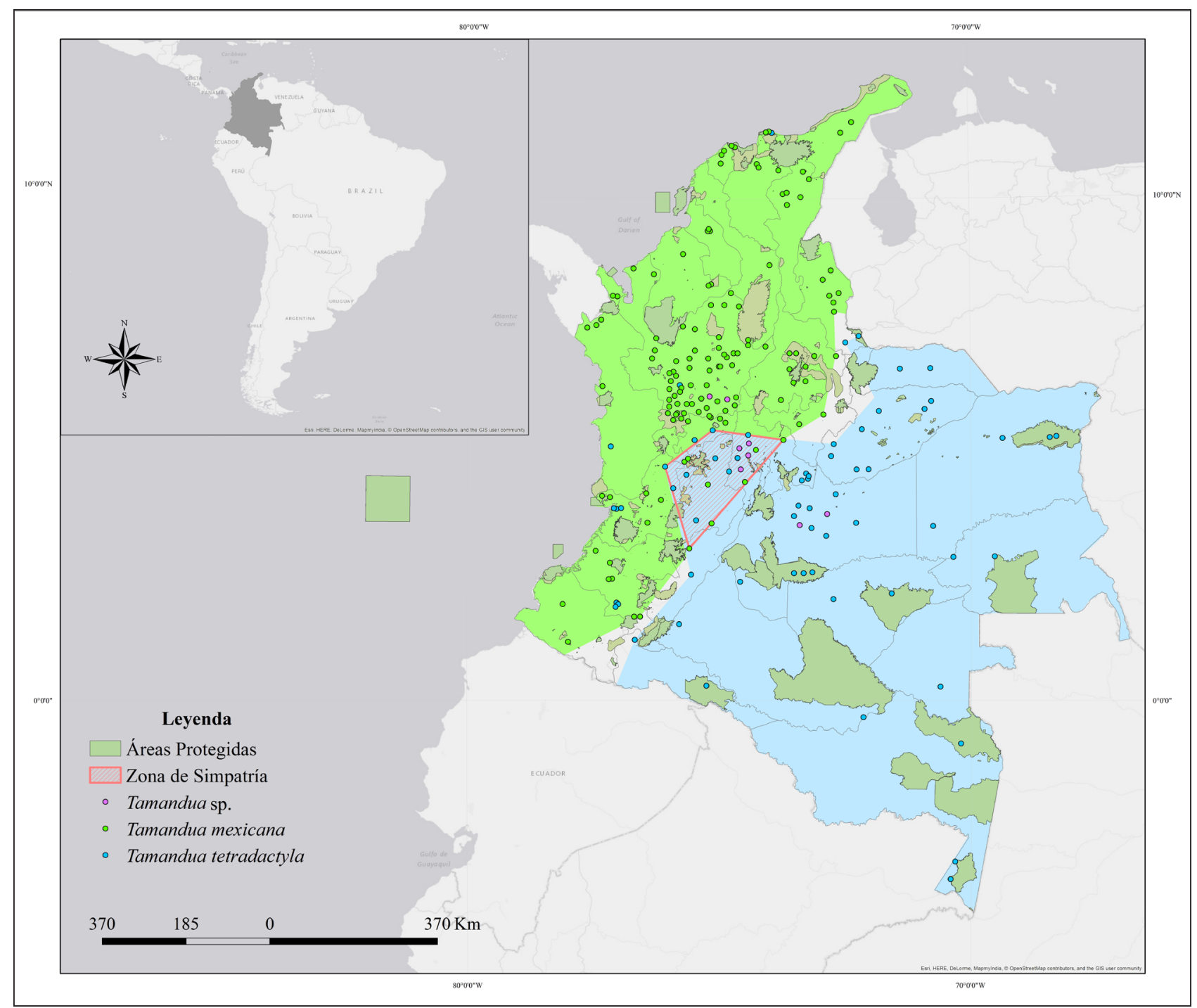

FIGURA 1. Distribución del género Tamandua y presencia en el sistema de áreas protegidas de Colombia. Círculos violeta: Tamandua sp.; círculos y sombreado verdes: T. mexicana; círculos y sombreado azules: T. tetradactyla. El polígono rosa marca la zona de simpatría entre las especies.

SINAP de Colombia, se pudo evidenciar la presencia del género en 10 Parques Nacionales Naturales (PNN), de los 59 existentes en el país. También se obtuvieron tres registros en Reservas Nacionales Naturales (RNN) y uno más en un Área de Importancia para la Conservación de Aves (AICAs). Tamandua mexicana presentó registros en el PNN Tayrona, Vía Parque Isla Salamanca, PNN Los Katíos, Serranía de San Lucas (AICA), Reserva Natural Bajo Cauca-Nechí y Área Natural Única Los Estoraques. Por su parte T. tetradactyla se registró en PNN El Tuparro, Tamá, Sierra de la Macarena, RNN Nukak, PNN Yaigojé Apaporis, La Paya y en la Reserva Natural Bosque de Yotoco. El área total de las áreas protegidas con presencia confirmada de T. mexicana fue de $2,024 \mathrm{~km}^{2}$; para T. tetradactyla el área total fue de $31,622.7 \mathrm{~km}^{2}$.

$\mathrm{Al}$ contrastar el mapa generado de la distribución de las especies en Colombia con los reportados por la UICN (2016) y AIUNAU \& Minambiente (2016), se pudo observar que existen ciertas coincidencias para las dos especies. Sin embargo, se encontraron registros no reportados en la literatura en la región de los Andes para T. tetradactyla en los departamentos de Cundinamarca, Huila, Caldas, Quindío y Valle del Cauca. Debido a estos registros se presenta una posible zona de simpatría en esta región (FIG. 1).

\section{Prioridades de investigación}

Se encontraron opiniones muy similares en la bibliografía y entrevistas a expertos en cuanto a las principales amenazas que enfrentan las especies de Tamandua en el país. La destrucción de hábitat, cacería, atropellamientos y el aislamiento de las poblaciones las afectan en mayor medida. Acorde a lo obtenido, las prioridades de investigación inmediatas para estas especies deben enfocarse a estudios sobre dinámica y tamaño poblacional y las amenazas a las que se ven expuestas, seguido por estudios ecológicos in situ y, por último, su sistemática en el país. 


\section{Discusıón}

La producción bibliográfica encontrada deja entrever la falta de información que existe actualmente en Colombia sobre las especies del género Tamandua. Con sólo siete documentos relacionados directamente con alguna de las especies y un manual de rehabilitación específico para todas las especies de hormigueros, no se poseen los datos suficientes para establecer aspectos de su ecología, como el estado y dinámica de sus poblaciones. Sin embargo, la información recopilada aportó datos significativos para establecer una distribución más precisa sobre el género, uno de los puntos más importantes al momento de realizar estudios ecológicos (Juliá et al., 2004). De igual forma, estos estudios ayudaron a establecer un orden de prioridades para investigación a nivel nacional.

En cuanto a los registros de distribución obtenidos, el género Tamandua presentó un patrón de distribución similar al mencionado por Rojano et al. (2014) y AIUNAU \& Minambiente (2016) en el cual T. mexicana se distribuye en el norte, occidente y centro del país y $T$. tetradactyla en el suroriente. No obstante, en el presente estudio se encontraron registros de presencia para ambas especies, tanto en colecciones biológicas como de avistamientos, que no se encontraban reportados con anterioridad en varios departamentos en la zona central del país. De acuerdo a estos registros puede existir una posible zona de simpatría en esta región, aunque no se sabe con seguridad si todos los individuos reportados fueron identificados correctamente, ya que las especies de este género son muy similares morfológicamente, por lo que podrían ocurrir confusiones al momento de su avistamiento. Sin embargo, se obtuvieron registros en algunos departamentos de esta zona, donde tres individuos de T. tetradactyla fueron capturados para su estudio y la identificación de la especie fue correcta. Es muy importante realizar estudios en estos departamentos que comprueben si existe la zona de simpatría y, si es así, determinar qué está causando la extensión en el rango de distribución de T. tetradactyla y su solapamiento con el de T. mexicana.

Por otro lado, se observa que la presencia confirmada del género en el SINAP abarca un 21,35\% de su área de distribución en el país. Esto sugiere que, por lo menos para T. tetradactyla $(20,2 \%)$, las zonas de protección ya establecidas están contribuyendo de forma significativa a la conservación de la especie. Muchos de los parques en los que se encontraron registros, como la Sierra de la Macarena, PNN Nukak y PNN Yaigojé-Apaporis, abarcan superficies considerables, están protegidos por las comunidades indígenas y, por lo tanto, son áreas de gran importancia para la conservación. Sin embargo, es importante mencionar que la mayoría de la superficie protegida está ubicada en Amazonia. En otros ecosistemas como la Orinoquia, especialmente en los departamentos de Casanare y Meta, las poblaciones de T. tetradactyla están prácticamente desprotegidas y constantemente amenazadas por las actividades humanas de esta región.

Siguiendo esta misma línea, el panorama para T. mexicana es completamente distinto. Al sólo tener el 1,15\% de su área de distribución dentro de zonas de protección, su presencia en el SINAP es muy baja, dejando así la gran mayoría de sus registros en zonas más intervenidas y fragmentadas. Por lo anterior, sería importante ampliar el rango de áreas protegidas existentes donde se obtuvo el mayor número de reportes, como es el caso de Vía Parque Isla Salamanca o el PNN Tayrona, dos de los lugares más importantes para la conservación de especies a nivel nacional. De igual forma se podrían crear nuevas áreas en los departamentos donde se obtuvieron varios reportes, como son Antioquia, Cundinamarca, Santander, Casanare y Meta, analizando la viabilidad de éstas y sus ecosistemas, para que así aumente el grado de conservación de las poblaciones de tamandúas en Colombia.

A pesar de que los resultados obtenidos en las entrevistas muestran que existe el interés necesario para promover un estado de conocimiento más amplio, hay pocos investigadores que se deciden aplicarlo y plasmarlo en estudios y / o proyectos. Uno de los principales problemas que ven los entrevistados es que estas especies no son fáciles de encontrar en su hábitat natural. Ortega Reyes et al. (2014) y la UICN (2016) plantean algo similar, por lo que estudios de densidad o estados poblacionales demandarían de importantes recursos económicos y dedicación. Uno de los primeros pasos para los investigadores interesados en trabajar con estas especies sería seleccionar las zonas con vacíos de información. Las zonas biogeográficas de la Amazonia y la Orinoquia serían ideales para realizar evaluaciones base que permitan establecer planes para efectuar monitoreos poblacionales, ya que es donde se encontró la menor cantidad de registros. Es muy importante promover los estudios en estos lugares y en zonas del país de las que se cuenta con un número mayor de registros, ya que podrían resolver diferentes inquietudes sobre la ecología de las tamandúas y su historia natural.

Actualmente las especies de tamandúas no se encuentran en una categoría de amenaza inmediata de extinción a nivel global, pero sí se ven afectadas como muchas otras por la destrucción del hábitat, la fragmentación, la cacería y los atropellamientos (Alves da Rosa, 2010; Superina et al., 2010; Ortega Reyes et al., 2014), algo que concuerda con los resultados obtenidos en este estudio. Debido a la poca información que existe para las especies de Tamandua, se desconoce el verdadero alcance que tienen estos problemas y presiones sobre sus poblaciones a nivel nacional. 


\section{AGRADECIMIENTOS}

A todos los investigadores y expertos nacionales e internacionales que se tomaron el tiempo para responder y ofrecer su información, principalmente a Tinka Plese y a Flavia Miranda por su constante interés y apoyo. A Amancay de Atacama Cepeda por su asesoría. Al Programa Nacional para la Conservación de Xenarthras en Colombia por darnos la oportunidad de mostrar este estudio y tenerlo en cuenta. A los museos e instituciones nacionales e internacionales, a las Corporaciones Autónomas Regionales y en general a todas esas personas que pusieron su granito de arena para que este estudio tomara el rumbo deseado y se cumplieran todos los objetivos.

\section{REFERENCIAS}

AIUNAU \& Minambiente. 2016. Programa nacional para la conservación y uso sostenible de las especies del superorden Xenarthra presentes en Colombia. Plan de acción 2014-2023. Ministerio de Medio Ambiente y Desarrollo Sostenible, Bogotá, Colombia. 56 pp.

Alberico, M., A. Cadena, J. Hernández-Camacho \& Y. Muñoz-Saba. 2000. Mamíferos (Synapsida: Theria) de Colombia. Biota Colombiana 1: 43-75.

Alves da Rosa, C., Q. Hobus \& A. Bager. 2010. Mammalia, Pilosa, Myrmecophagidae, Tamandua tetradactyla (Linnaeus, 1758): Distribution extension. Check List 6: 52-53. https: / / doi.org/10.15560/6.1.052

Ayerbe-Quiñones, F., H. E. Ramírez-Chaves, O. Mejía-Egas \& A. Castillo-Díaz. 2006. Plan de ordenamiento y manejo de la subcuenca Sambingo - Hato Viejo, municipios de Bolívar, Mercaderes y Florencia, Cauca, Colombia. Fundación Mamaskato - Corporación Autónoma Regional del Cauca, Popayán, Cauca, Colombia. 62 p.

Balaguera-Reina, S. A., J. F. González-Maya \& A. Acero. 2010. Fauna nocturna asociada a los manglares y otros humedales en la Vía Parque Isla Salamanca, departamento del Caribe colombiano. Boletín de Investigaciones Marinas y Costeras 1: 191-231.

Castaño, J. H. \& J. D. Corrales. 2010. Mamíferos de la cuenca del Rio La Miel (Caldas): diversidad y uso cultural. Boletín Científico del Museo de Historia Natural 14: 56-75.

Castaño, J. H., Y. Muñoz-Saba, J. E. Botero \& J. H. Vélez. 2003. Mamíferos del departamento de Caldas. Biota Colombiana 4: 247-259.

Castaño-Uribe C., J. F. González-Maya, C. Ange, S. A. Balaguera-Reina, A. Cepeda, D. A. ZárrateCharry \& I. C. Cepeda. 2010. Profundización de la caracterización y diagnóstico de las poblaciones de félidos y otros mamíferos medianos y grandes en zonas prioritarias de conservación en el canal del dique sector Sucre: aproximación al manejo sostenible de unidades productivas utilizando modelos de especies sombrilla. Informe Técnico Final. Fundación Herencia Ambiental Caribe-Conservación InternacionalCARSUCRE, Sincelejo, Sucre. 115 pp.

CORPOCHIVOR. 2015. Plan de manejo ambiental del distrito regional de manejo integrado Cuchillas Negras y Guanque. Proyecto 201, protección, recuperación y manejo de la biodiversidad y los ecosistemas estratégicos. Corporación Autónoma Regional de Chivor, Huila, Colombia. $260 \mathrm{pp}$.

Cruz, D. R. \& P. A. Ramos. 2011. Cambio cultural, económico e institucional: Análisis de la sostenibilidad de la actividad de la cacería en la comunidad de la Ceiba, Rio Inírida, Guainía, Colombia. Tesis de pregrado. Facultad de Estudios Ambientales y Rurales, Ecología, Universidad Javeriana, Bogotá. 170 pp.

Cuartas-Calle, C. A. \& J. Muñoz-Arango. 2003. Listado de mamíferos (Mammalia: Theria) del departamento de Antioquia, Colombia. Biota Colombiana 4: 65-78.

Cuesta-Ríos, E. Y., J. D. Valencia-Mazo \& A. M. Jiménez-Ortega. 2007. Aprovechamiento de los vertebrados terrestres por una comunidad humana en los bosques tropicales (Tutunendo, Chocó, Colombia). Revisa Institucional Universidad Tecnológica del Chocó: Investigación, Biodiversidad y Desarrollo 26: 37-43.

De Armas-Mendoza, E. \& G. Forero-Medina. 2007. Levantamiento preliminar de medianos y grandes mamíferos no voladores en dos localidades del municipio de Arauca (Colombia). Tesis de Pregrado, Universidad Nacional de Colombia, Sede Orinoquia, Arauca. 29 pp.

De La Ossa-V., J. \& S. Galván-Guevara. 2015. Registro de mortalidad de fauna silvestre por colisión vehicular en la carretera Toluviejo - Ciénaga La Caimanera, Sucre, Colombia. Biota Colombiana 16: 67-77.

Defler, T. \& J. V. Rodríguez-Mahecha. 1998. La fauna de la Orinoquía. 135 - 156 pp. En: Colombia Orinoco ( D. Fajardo-Montaña, ed.). Fondo para la Protección del Medio Ambiente José Celestino Mutis, FEN Colombia, Bogotá, Colombia. 324 pp.

Etter, A. 2001. Puinawai y Nukak: Caracterización ecológica general de dos Reservas Nacionales Naturales de la Amazonia colombiana. Pontificia Universidad Javeriana, IDEADE, Bogotá. 382 pp. 
Ferrer-Pérez, A., M. Beltrán-Gutiérrez \& C. A. Lasso. 2009. Mamíferos de la estrella fluvial de Inírida: ríos Inírida, Guaviare, Atabapo, Orinoco. Biota Colombiana 10: 209-218.

Galván-Guevara, S. 2010. Mamíferos y aves silvestres registradas en una zona de los Montes de María, Coloso, Sucre, Colombia. Revista Colombiana de Ciencia Animal 2: 45-57.

Gardner, A. 2008. Mammals of South America. Volume 1: Marsupials, xenarthrans, shrews, and bats. University of Chicago Press, Chicago. 669 pp. https://doi.org/10.7208/ chicago/9780226282428.001.0001

Gomez, L. L., J. A. Benavides, M. Mayor-Jaramillo, C. Carlos \& N. C. Mesa. 2010. Amblyomma nodosum (Acari: Ixodidae) en Tamandua tetradactyla (Pilosa: Vermilingua)dela reservabosqueYotoco, departamento del Valle del Cauca, Colombia. Memorias, I Congreso Latinoamericano (IV Argentino) de Conservación de la Biodiversidad, San José de Tucumán, Argentina.

Humanez-López, E., J. Chacón-Pacheco \& T. Plese. 2015. Área de extracción de xenartros en el Caribe colombiano. Edentata 16: 65-68.

Juliá, J. P., E. Richard \& J. Samaniego. 2004. Notas sobre la distribución geográfica del oso melero (Tamandua tetradactyla, Xenarthra: Myrmecophagidae) en el noroeste Argentino. Nótulas Faunísticas 66: 1-4.

Meisel, J. E. 2003. Tamandua: A vested interest in ants. Ceiba Foundation for Tropical Conservation (CFTC). <http: / / www.ceiba.org/articles/tamandua.htm>. Consultada 24 de marzo del 2011.

Miranda, F., A. Fallabrino, M. C. Arteaga, D. G. Tirira, D. A. Meritt \& M. Superina. 2014. Tamandua tetradactyla. The IUCN Red List of Threatened Species 2014. <http://www.iucnredlist.org>. Consultada 15 de agosto de 2015.

Ortega Reyes, J., D. G. Tirira, M. C. Arteaga \& F. Miranda. 2014. Tamandua mexicana. The IUCN Red List of Threatened Species. 2014. <http:/ / www.iucnredlist.org $>$. Consultada 15 de agosto de 2015.

Padilla-Cordero, H. D., C. Rojano-Bolaño, E. D. Ramos-Espitia \& A. G. Alvarez-Otero. 2014. Parámetros hematológicos del Oso Hormiguero (Tamandua mexicana) en vida libre en Colombia. II Congreso Colombiano de Patógenos y Huéspedes en Vida Silvestre, Cartagena, Colombia.

Polanco-Ochoa, R., V. Jaimes \& W. Piragua. 1999. Los mamíferos del Parque Nacional Natural La Paya, Amazonía colombiana. Revista de la Academia Colombiana de Ciencias Exactas, Físicas y Naturales 23: 671-682.

Ramírez-Chaves, H. E. \& E. A. Noguera-Urbano. 2010. Lista preliminar de los mamíferos (Mammalia: Theria) del departamento de Nariño, Colombia. Biota Colombiana 11: 117-140.

Ramírez-Chaves, H. E., F. Ayerbe-Quiñones \& O. Mejía-Egas. 2010. Mamíferos de la cuenca alta del Río Patía en el departamento del Cauca, Colombia. Boletín Científico del Museo de Historia Natural 14: 92-113.

Rendón, L. M., F. Guhl, J. M. Cordovez\&E.Erazo. 2015. New scenarios of Trypanosoma cruzi transmission in the Orinoco region of Colombia. Memorias do Instituto Oswaldo Cruz 110: 283-288. https: / / doi.org/10.1590 / 0074-02760140403

Rojano, C., H. Padilla, E. Almentero \& G. Alvarez. 2013. Percepciones y usos de los Xenarthra e implicaciones para su conservación en Pedraza, Magdalena, Colombia. Edentata 14: 58-65. https: / doi.org/10.5537 / 020.014.0107

Rojano, C., L. Miranda y R. Ávila. 2014. Manual de rehabilitación de hormigueros de Colombia. Fundación Cunaguaro, Geopark Colombia S.A.S., El Yopal, Colombia. 155 pp.

Rojano-Bolaño, C., L. Miranda \& R. Ávila. 2015. Endoparásitos de Myrmecophaga tridactyla y Tamandua tetradactyla (Pilosa: Vermilingua) silvestres en Casanare, Colombia. Revista Colombiana de Ciencia Animal 7: 154-157.

Romero M., G. Galindo, J. Otero \& D. Armenteras. 2004. Ecosistemas de la cuenca del Orinoco colombiano. Instituto de Investigaciones de Recursos Biológicos Alexander von Humboldt, Bogotá. 189 pp.

Sánchez, F., P. Sánchez-Palomino \& A. Cadena. 2004. Inventario de mamíferos en un bosque de los Andes centrales de Colombia. Caldasia 26: 91-309.

Sandoval-Gómez, V., H. R. Ramírez-Chaves \& D. Marín. 2012. Registros de hormigas y termitas presentes en la dieta de osos hormigueros (Mammalia: Myrmecophagidae) en tres localidades de Colombia. Edentata 13: 1-9. https: / / doi.org/10.5537/020.013.0104

Solari, S., Y. Muñoz-Saba, J. V. Rodríguez-Mahecha, T. R. Defler, H. E. Ramírez-Chaves \& F. Trujillo. 2013. Riqueza, endemismo y conservación de los mamíferos de Colombia. Mastozoología Neotropical 20: 301-365.

Superina, M., T. Plese, N. Moraes-Barros \& A. M. Abba. 2010. The 2010 anteater Red List assessment. Edentata 11: 96-114. https://doi. org / 10.5537/020.011.0201 
Torres-Mejía, A. M. \& J. de la Fuente. 2006. Risks associated with ectoparasites of wild mammals in the department of Quindío, Colombia. International Journal of Applied Research in Veterinary Medicine 3: 187-192.

Triana-Ardila, S., S. Álvarez \& D. Lizcano. 2011. Dieta de oso hormiguero (Tamandua mexicana) en el Área Natural Única los Estoraques Norte de Santander, Colombia. Tesis de pregrado, Universidad de Pamplona, Norte de Santander, Colombia. $45 \mathrm{pp}$.

UICN. 2016. The IUCN Red List of Threatened Species. International Union for Conservation of Nature and Natural Resources. IUCN Red List of Threatened Species. <http://www.iucnredlist.org $>$. Consultada 10 de julio de 2016.

Vásquez, C., S. Robledo, J. Calle \& O. Triana. 2013. Identificación de nuevos escenarios epidemiológicos para la enfermedad de Chagas en la región momposina, norte de Colombia. Biomédica 33: 536-537. https://doi.org/10.7705/biomedica. v33i4.836

Vega-Orduz, L. F. 2007. Mamíferos de Casanare: una aproximación a la riqueza de especies y los ecosistemas donde habitan. Informe final. Dirección Técnica de Medio Ambiente, Secretaría de Agricultura, Ganadería y Medio Ambiente, Gobernación de Casanare, El Yopal, Colombia. 56 pp.

Wetzel, R. M. 1985. The identification and distribution of recent Xenarthra (= Edentata) (G. G. Montgomery, ed.). Pp. 5-22 in: The evolution and ecology of armadillos, sloths, and vermilinguas. Smithsonian Institution Press, Washington and London.

Recibido: 28 de septiembre de 2016; Aceptado: 3 de diciembre de 2016

\section{APÉNDICE 1. INVESTIGADORES ENTREVISTADOS}

Diego J. Lizcano - Biólogo colombiano - Visiting scientist, Conservation Internacional - Sociedad Colombiana de Mastozoología

German Darío Gómez - Biólogo/Magister colombiano - Profesor Universidad del Quindío, Director Reserva Natural Montaña del Ocaso - Asociación Colombiana de Zoología

Hugo López Arévalo - Biólogo colombiano - Profesor asistente Instituto de Ciencias Naturales Universidad Nacional

Iván Mauricio Vela Vargas - Biólogo/Magister colombiano - Investigador asociado a ProCat, estudiante de maestría

Iván Ruiz - Biólogo costarricense - Coordinador de investigación

Luisa Fernanda Vega-Orduz - Bióloga/Magister colombiana, estudiante de doctorado

Silvia J. Álvarez - Bióloga colombiana

Stephanie C. Triana-Ardila - Bióloga colombiana

Tinka Plese - MSc - Directora Fundación AIUNAU 